\title{
Discovery of diffuse radio emission at the center of the most X-ray-Iuminous cluster RX J1347.5-1145
}

\author{
M. Gitti ${ }^{1}$, C. Ferrari ${ }^{2}$, W. Domainko ${ }^{3}$, L. Feretti ${ }^{4}$, and S. Schindler ${ }^{2}$ \\ 1 INAF - Osservatorio Astronomico di Bologna, via Ranzani 1, 40127 Bologna, Italy \\ e-mail: myriam.gitti@oabo.inaf.it \\ 2 Institut für Astro- und Teilchen Physik, Universität Innsbruck, Technikerstraße 25, 6020 Innsbruck, Austria \\ 3 Max-Planck Institute for Nuclear Physics, Saupfercheckweg 1, 69117 Heidelberg, Germany \\ 4 INAF - Istituto di Radioastronomia, via Gobetti 101, 40129 Bologna, Italy
}

Received 17 April 2007 / Accepted 8 June 2007

ABSTRACT

\begin{abstract}
Context. We report on new VLA radio observations of the distant cluster RX J1347.5-1145, which is the most luminous in X-rays. Aims. We aim at investigating the possible presence of diffuse and extended radio emission in this very peculiar system which shows both a massive cooling flow and merging signatures.

Methods. New low resolution $(\sim 18$ arcsec) VLA radio observations of this cluster are combined with higher resolution $(\sim 2$ arcsec $)$ data available in the VLA archive.

Results. We discover the presence of a diffuse and extended $(\sim 500 \mathrm{kpc})$ radio source centered on the cluster, unrelated to the radio emission of the central AGN. The properties of the radio source, in particular a) its occurrence at the center of a massive cooling flow cluster, b) its total size comparable to that of the cooling region, c) its agreement with the observational trend between radio luminosity and cooling flow power, indicate that RX J1347.5-1145 hosts a radio mini-halo. We suggest that the radio emission of this mini-halo, which is the most distant object of its class discovered up to now, is due to electron re-acceleration triggered by the central cooling flow. However, we also note that the morphology of the diffuse radio emission shows an elongation coincident with the position of a hot subclump detected in X-rays, thus suggesting that additional energy for the electron re-acceleration might be provided by the submerger event.
\end{abstract}

Key words. galaxies: clusters: individual: RX J1347.5-1145 - radiation mechanisms: non-thermal galaxies: intergalactic medium - galaxies: cooling flows - acceleration of particles - radio continuum: galaxies

\section{Introduction}

Diffuse and extended synchrotron emission has been detected in a number of galaxy clusters, revealing the presence of relativistic electrons and magnetic fields diffused into the intra-cluster medium (ICM). These radio sources, characterized by steep radio spectra and sizes of $\sim 1 \mathrm{Mpc}$, are usually classified as "radio halos" when permeating the cluster center, "radio relics" when located in the cluster external regions (e.g., Feretti \& Giovannini 2007). The presence of both halos and relics in galaxy clusters have been suggested to be strongly connected to the existence of cluster-cluster mergers, which would provide the energy for reaccelerating the electrons to ultra-relativistic energies (primary models; e.g., Brunetti et al. 2001). Relativistic electrons could also result from inelastic nuclear collisions between relativistic protons and thermal ions of the ICM (secondary models; e.g. Blasi \& Colafrancesco 1999). Up to now, the comparison of observational and analytical results is in favor of primary models related to cluster mergers (e.g., Brunetti 2003; Blasi et al. 2007). In particular, radio halos and relics are not commonly seen in clusters characterized by short central cooling time and high central brightness peak, the so-called cooling flow clusters (e.g., Fabian 1994; Peterson \& Fabian 2006), since the cooling flow is likely suppressed or disrupted by a major merger (Gomez et al. 1997).

The central regions of cooling flow clusters host an exceptional class of diffuse radio sources, called "mini-halos" due to their smaller extension $(\sim 500 \mathrm{kpc})$. They usually surround a powerful radio galaxy. However, their radio emission is not due to radio lobes fed by an active galactic nucleus (AGN), but, again, to the presence of relativistic particles and magnetic field in the ICM. Gitti et al. (2002) proposed that the synchrotron emission from radio mini-halos could be due to a relic population of (primary) relativistic electrons re-accelerated by MHD turbulence, with the necessary energy supplied by the cooling flow process itself. Alternatively, Pfrommer \& Enßlin (2004) discussed the possibility of radio emission from secondary electrons. The successful application of the re-acceleration model to two cooling flow clusters (Gitti et al. 2002, 2004) has given support to a primary origin of the relativistic electrons radiating in radio mini-halos, although detailed observations of a large sample are required to understand the origin of these sources.

RX J1347.5-1145 is the most X-ray-luminous cluster discovered to date $\left(L_{\mathrm{X}}=6 \times 10^{45} \mathrm{erg} \mathrm{s}^{-1}\right.$ in the [2-10] keV energy range, Schindler et al. 1995; Gitti \& Schindler 2004). It shows a very peaked surface brightness profile and hosts a massive cooling flow in its center (Gitti \& Schindler 2004). Submillimeter and millimeter observations in its direction detected significant Sunyaev-Zeldovich (SZ) signal and revealed complex morphological and temperature structures of the cluster region (Komatsu et al. 1999, 2001; Pointecouteau et al. 1999, 2001). Recent Chandra (Allen et al. 2002) and XMM-Newton (Gitti \& Schindler 2004) observations identified a region with hot, bright 
X-ray emission located $\sim 20$ arcsec to the main X-ray surface brightness peak in the south-east direction, at a position coincident with the region of enhanced SZ effect (Komatsu et al. 2001). These results were interpreted as indications of a subcluster merger, making RX J1347.5-1145 a very peculiar system with a complex dynamical evolution. If on the one hand there is indication of a disturbed dynamical state in the south-east region, on the other hand the strong cooling flow points to a relatively long interval of time in which the cluster has evolved undisturbed to a relaxed state (Gitti et al. 2007). This cluster is thus perfectly suited to study the interplay of massive cooling flows and subcluster mergers, which are very rarely observed in the same system, on the re-acceleration of relativistic electrons. The radio emission in the central $3 \times 3 \mathrm{arcmin}^{2}$ of RX J1347.5-1145, as seen from the NRAO VLA Sky Survey (NVSS; Condon et al. 1998), shows a strong central source and some hints of a possible extended emission. In this Letter we present the results from new high sensitivity $1.4 \mathrm{GHz}$ radio observations of this cluster, which led to the discovery of diffuse radio emission that may be classified as a mini-halo.

RX J1347.5-1145 (hereafter RX J1347) is at a redshift of $0.451^{1}$, thus being the most distant cluster in which a radio mini-halo has been detected.

\section{Observations and data reduction}

We performed Very Large Array ${ }^{2}$ observations of the radio source RX J1347 at $1.4 \mathrm{GHz}$ in C-configuration, that we combined with same and higher resolution (VLA in A-configuration) observations at the same frequency available in the VLA archive (see Table 1 for details regarding these observations). In all observations the source 3C 286 is used as the primary flux density calibrator, while the source $1351-148$ is used as the secondary phase calibrator. Data reduction is done using the NRAO AIPS (Astronomical Image Processing System) package. Accurate editing of the uv data is applied to identify and remove bad data. Images are produced by following the standard procedures: Calibration, Fourier-Transform, Clean and Restore. Self-calibration is applied to remove residual phase variations. Data in array $\mathrm{C}$ from the two different observations are reduced separately, in order to analyze the possible existence of spurious features, and the resulting images are then combined. The final images, produced using the AIPS task IMAGR, show the contours of the total intensity (Figs. 1 and 2).

\section{Results}

Figure 1 shows the radio map of RX J1347 observed at $1.4 \mathrm{GHz}$ with the VLA in $\mathrm{C}$ configuration, with a restoring beam of $17.8^{\prime \prime} \times 13.6^{\prime \prime}$. The source is clearly extended with a strong central component and an amorphous morphology elongated toward the south (S). The total size is $\sim 107^{\prime \prime}(620 \mathrm{kpc})$ in the northsouth (NS) direction and the total flux density is $55.3 \pm 0.6 \mathrm{mJy}$ (see Table 2). The source visible to the south-west (SW) of the extended emission is associated to a cluster galaxy, and has a total flux density of $4.50 \pm 0.06 \mathrm{mJy}$.

Figure 2 shows the radio map of the very central field of RX J1347 observed at $1.4 \mathrm{GHz}$ with the VLA in A configuration,

${ }^{1}$ With a Hubble constant of $H_{0}=70 \mathrm{~km} \mathrm{~s}^{-1} \mathrm{Mpc}^{-1}$, and $\Omega_{\mathrm{M}}=$ $1-\Omega_{\Lambda}=0.3$, the luminosity distance is $2506 \mathrm{Mpc}$ and the angular scale is $5.77 \mathrm{kpc} \operatorname{arcsec}^{-1}$.

2 The Very Large Array (VLA) is a facility of the National Radio Astronomy Observatory (NRAO). The NRAO is a facility of the National Science Foundation, operated under cooperative agreement by Associated Universities, Inc.
Table 1. VLA data.

\begin{tabular}{ccccc}
\hline \hline $\begin{array}{c}\text { Observation } \\
\text { date }\end{array}$ & $\begin{array}{c}\text { Frequency } \\
(\mathrm{MHz})\end{array}$ & $\begin{array}{c}\text { Bandwidth } \\
(\mathrm{MHz})\end{array}$ & Array & $\begin{array}{c}\text { Exp. Time } \\
(\mathrm{h})\end{array}$ \\
\hline Sep.-2005 & $1365 / 1435$ & 50.0 & $\mathrm{C}$ & 2.0 \\
Dec.-1998 & $1385 / 1465$ & 25.0 & $\mathrm{C}$ & 2.0 \\
Apr.-1998 & $1385 / 1465$ & 25.0 & $\mathrm{~A}$ & 3.0 \\
\hline
\end{tabular}

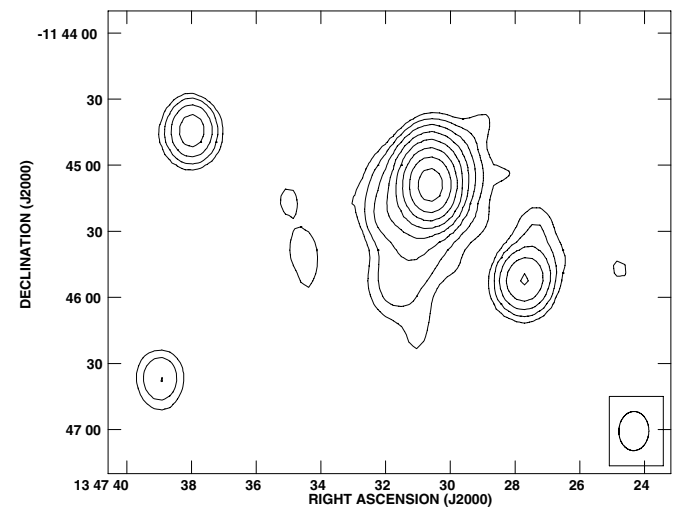

Fig. 1. 1.4 GHz VLA map of RX J1347 at a resolution of $17.8^{\prime \prime} \times$ $13.6^{\prime \prime}$ (the beam is shown in the lower-right corner). The rms noise is $0.04 \mathrm{mJy} / \mathrm{beam}$. The first contour corresponds to $5 \sigma$, the ratio between two consecutive contours being 2 .

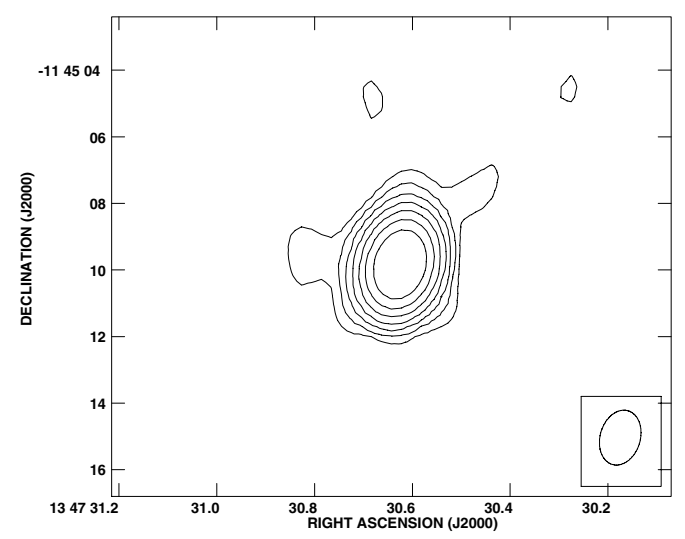

Fig. 2. 1.4 GHz VLA map of RX J1347 at a resolution of $1.7^{\prime \prime} \times$ $1.2^{\prime \prime}$ (the beam is shown in the lower-right corner). The rms noise is $0.03 \mathrm{mJy} /$ beam. The first contour corresponds to $5 \sigma$, the ratio between two consecutive contours being 2 .

with a restoring beam of $1.7^{\prime \prime} \times 1.2^{\prime \prime}$. With this high resolution map it is possible to image the central source and to spot the presence of discrete sources that can contribute to the total flux and extended morphology detected at lower resolution. The central source, located at RA(J2000): $13^{\mathrm{h}} 47^{\mathrm{m}} 30.6^{\mathrm{s}}$, $\operatorname{Dec}(\mathrm{J} 2000):-11^{\circ} 45^{\prime} 09.9^{\prime \prime}$, is resolved, showing hints of faint structures (detected at a $6 \sigma$ level) emanating to the east (E) and north-west (NW) directions from the center. No relevant structures are visible toward the $\mathrm{S}$ direction. The total flux density is $29.8 \pm 0.3 \mathrm{mJy}$. The high resolution structure of the central source strongly favours the possibility that it is unrelated to the diffuse emission detected at lower resolution. In this high resolution map the source to the $\mathrm{SW}$, located at RA(J2000): $13^{\mathrm{h}} 47^{\mathrm{m}} 27.7^{\mathrm{s}}$, $\operatorname{Dec}(\mathrm{J} 2000):-11^{\circ} 45^{\prime} 53.4^{\prime \prime}$ (not visible in Fig. 2), has a total flux density of $1.90 \pm 0.04 \mathrm{mJy}$. Its morphology suggests that it is a head-tail radio galaxy. The difference in the fluxes measured at 


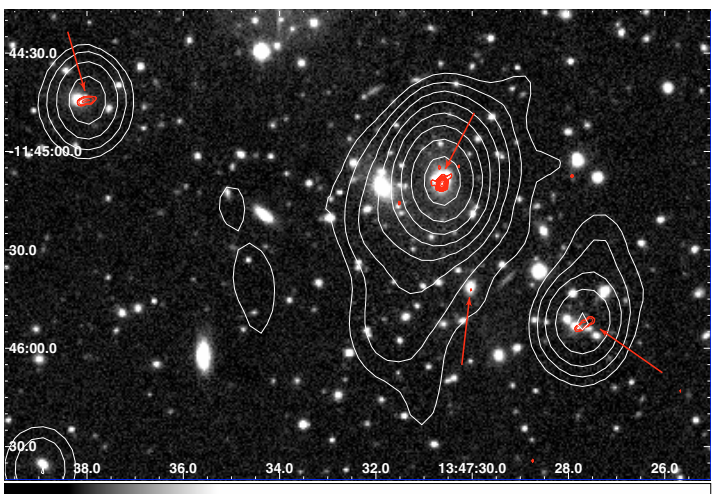

Fig. 3. Optical I-band image of the central field of RX J1347. Overlayed are the $1.4 \mathrm{GHz}$ radio contours at low (white) and high (red) resolution. The beam, rms noise and contours are the same as in Figs. 1 and 2, respectively. The arrows show the discrete radio sources identified for the subtraction from the low resolution radio map.

high and low resolution is thus due to the diffuse emission associated to the tail of the radio source.

In order to put in evidence the diffuse emission, it is essential to subtract the contribution of discrete radio sources. In a conservative way, they are identified as the radio sources detected in the high resolution map at $5 \sigma$ level, having obvious association with an optical galaxy. The four sources selected with this method are indicated by red arrows in Fig. 3. The clean components of the discrete sources are restored with a circular beam of $18^{\prime \prime}$ and then subtracted from the low resolution map convolved with the same beam. This procedure allows us to estimate the total flux density of the diffuse radio emission $\left(S_{1.4 \mathrm{GHz}}=25.2 \pm 0.3 \mathrm{mJy}\right)$ and derive its surface brightness map, which is shown in Fig. 4 overlayed onto the $\mathrm{X}$-ray image. The size of the diffuse radio emission $(\sim 435 \times 600 \mathrm{kpc})$ is comparable to that of the cooling region ( $420 \mathrm{kpc}$, Gitti et al. 2007), thus indicating that the diffuse radio source may be classified as a mini-halo. We also note that the morphology of the radio emission shows an elongation coincident with the position of the hot SE subclump detected in X-rays (Allen et al. 2002; Gitti \& Schindler 2004). Additionally, the SE excess in the ICM X-ray brightness and temperature radial profiles corresponds to a clear excess in the radio surface brightness of the diffuse radio source (see Fig. 5). The excess $\mathrm{X}$-ray bolometric luminosity of the SE quadrant between radii of $\sim 10^{\prime \prime}$ and $\sim 35^{\prime \prime}$, where the subclump is most apparent at the Chandra resolution, corresponds to $\sim 5 \%$ of the total cluster luminosity (Allen et al. 2002). Interestingly, we estimated that the excess radio flux of the SE quadrant in the region where the enhancement is most evident in the radio surface brightness profile (see Fig. 5, lower panel) corresponds to $\sim 5 \%$ of the total radio flux density of the mini-halo.

The main radio results concerning the central source are summarized in Table 2, where we also report the monochromatic radio power at each frequency calculated as $P_{v}=4 \pi D_{\mathrm{L}}^{2} S_{v}$ (here $D_{\mathrm{L}}$ is the luminosity distance and $S_{v}$ is the flux density at the frequency $v$ ).

\section{Discussion and conclusions}

As already discussed in the Introduction, RX J1347 is a peculiar cluster. It hosts a massive cooling flow and shows a disturbed dynamical state in the SE quadrant. Furthermore, it is dominated by two Brightest Cluster Galaxies (BCGs). If on the one hand this is unusual for massive cooling flow clusters, on the other hand based on its optical spectrum the western BCG is an AGN, with

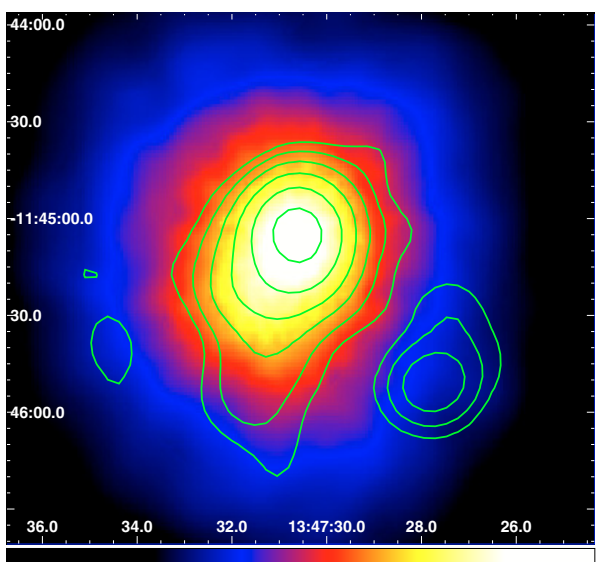

Fig. 4. Adaptively smoothed $X M M$-Newton image of the central region of RX J1347 (data from Gitti \& Schindler 2004). A bright subclump is visible $\sim 20^{\prime \prime}$ to the SE of the center (see also Allen et al. 2002). Overlayed are the $1.4 \mathrm{GHz}$ contours of the diffuse radio emission at a resolution of $18^{\prime \prime} \times 18^{\prime \prime}$ after the subtraction of the discrete radio sources identified at higher resolution (see Fig. 3). The rms noise is $0.05 \mathrm{mJy} / \mathrm{beam}$. The first contour corresponds to $5 \sigma$, the ratio between two consecutive contours being 2 .

typical emission lines of giant ellipticals at the center of cooling flow clusters (Cohen \& Kneib 2002). The presence of two dominant galaxies is probably related to a submerger event, since the giant ellipticals observed in the center of the cluster could be the BCGs of the two colliding subclusters. Indications of a recent or ongoing submerger event come also from the detection of a hot X-ray subclump in the SE quadrant (Allen et al. 2002; Gitti \& Schindler 2004). Interestingly, we detect diffuse radio emission at the cluster center. Its occurrence in a massive cooling flow cluster and its total size comparable to that of the cooling region ( $\sim 20 \mathrm{kpc}$, Gitti et al. 2007) indicate that the diffuse radio source may be classified as a mini-halo.

Gitti et al. (2002) developed a theoretical model which accounts for the origin of radio mini-halos as related to electron reacceleration by MHD turbulence in cooling flows. In this model, the necessary energetics to power radio mini-halos is supplied by cooling flows themselves, through the compressional work done on the ICM and the frozen-in magnetic field. This supports a direct connection between cooling flows and radio mini-halos. A full application of the model would be necessary to test its consistency with the observational properties of RX J1347, as done for the mini-halos in Perseus (Gitti et al. 2002), and A2626 (Gitti et al. 2004). Unfortunately, this is not possible with the present data because information on the spectral index properties, essential to test two out of the three observational constraints predicted by the model (namely, the brightness profile, the total radio spectrum and the radial spectral steepening, Gitti et al. 2002), is missing. Here, we can test qualitatively the consistency of the observational X-ray and radio properties of RX J1347 with some correlation predicted by the model. In particular, since the re-acceleration model assumes a connection between the origin of the synchrotron emission and the cooling flow, a trend between the radio power on the mini-halos and the maximum power of the cooling flows is expected. This has been observed in a first sample of mini-halos selected by Gitti et al. (2004). By plotting the integrated radio luminosity $v P_{v}$ versus the maximum power of the cooling flow $P_{\mathrm{CF}}=\dot{M} k T / \mu m_{\mathrm{p}}$ (here $\dot{M}$ is the mass accretion rate, $k$ the Boltzmann constant, $T$ the ICM temperature, $\mu \approx 0.61$ the molecular weight, and $m_{\mathrm{p}}$ the proton mass), these authors found that the strongest radio mini-halos are 
Table 2. Radio results for RX J1347. The size is estimated in the EW-NS directions along the maximum extent of the source.

\begin{tabular}{ccccccc}
\hline \hline Map & $\begin{array}{c}\text { Beam } \\
\left({ }^{\prime \prime 2}\right)\end{array}$ & $\begin{array}{c}\text { rms } \\
(\mathrm{mJy} / \mathrm{beam})\end{array}$ & $\begin{array}{c}\text { Size } \\
\left({ }^{2}\right)\end{array}$ & $\begin{array}{c}\text { Peak } \\
(\mathrm{mJy} / \mathrm{beam})\end{array}$ & $\begin{array}{c}\text { Total flux } \\
(\mathrm{mJy})\end{array}$ & $\begin{array}{c}\text { Radio power } \\
\left(10^{24} \mathrm{~W} \mathrm{~Hz}^{-1}\right)\end{array}$ \\
\hline array C & $17.8 \times 13.6$ & 0.04 & $73.0 \times 107.4$ & $37.8(0.4)$ & $55.3(0.6)$ & $41.5(0.5)$ \\
array A & $1.7 \times 1.2$ & 0.03 & $6.8 \times 5.1$ & $27.6(0.3)$ & $29.8(0.3)$ & $22.4(0.2)$ \\
subtracted & $18.0 \times 18.0$ & 0.05 & $75.6 \times 103.7$ & $11.0(0.1)$ & $25.2(0.3)$ & $18.9(0.2)$ \\
\hline
\end{tabular}

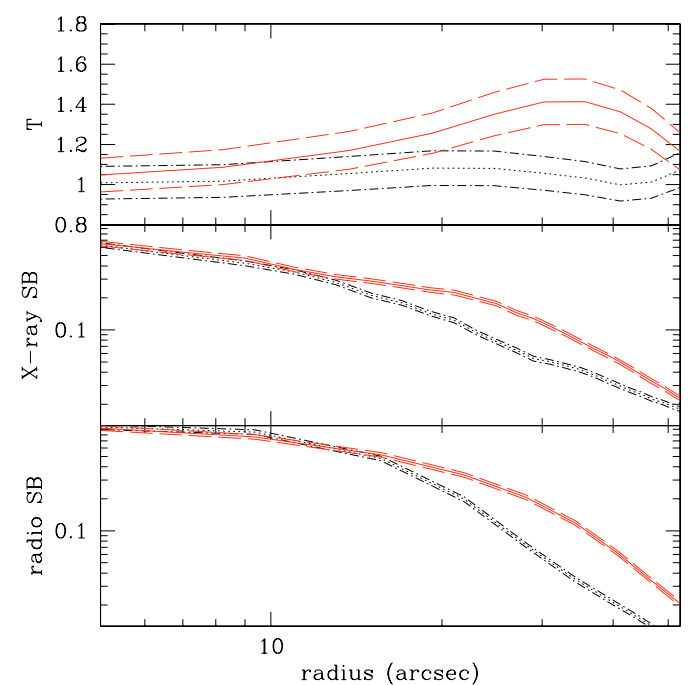

Fig. 5. The radial profiles extracted along the SE direction including the subclump ( $40^{\circ}$ east of south), which are shown with the solid lines, are compared to the radial profiles extracted along the opposite direction $\left(40^{\circ}\right.$ west of north), shown with the dotted lines. The profiles (embedded in the $1 \sigma$ error envelopes shown with thin dashed and dot-dashed lines, respectively) are normalized with respect to the central values. Clear enhancements are visible in all profiles along the SE direction. Upper panel: temperature profile derived from the XMM-Newton hardness ratio map presented by Gitti \& Schindler (2004, Fig. 4). Middle panel: X-ray surface brightness profile derived from XMM-Newton data (Gitti \& Schindler 2004). Lower panel: radio surface brightness profile of the mini-halo derived from the map shown in Fig. 3.

associated with the most powerful cooling flows. In more convenient units, the expression for the cooling flow power can be rewritten as: $P_{\mathrm{CF}} \sim 10^{41}\left(\dot{M} / 1 M_{\odot} \mathrm{yr}^{-1}\right)(k T / 1 \mathrm{keV}) \mathrm{erg} \mathrm{s}^{-1}$. We estimate this quantity from the XMM-Newton data presented by Gitti \& Schindler (2004). To be consistent with the mini-halo sample data collected from literature (Gitti et al. 2004), we adopt the overall cluster temperature $k T=10.0 \pm 0.3 \mathrm{keV}$ (Gitti \& Schindler 2004) and mass accretion rate $\dot{M}=1600_{-650}^{+140} M_{\odot} \mathrm{yr}^{-1}$, derived by fitting the central spectrum with a standard cooling flow model.

In Fig. 6 we show with a triangle the radio power $\left[v P_{v}\right]_{1.4 \mathrm{GHz}}=(2.65 \pm 0.03) \times 10^{41} \mathrm{erg} \mathrm{s}^{-1}$ and cooling flow power $P_{\mathrm{CF}}=1.6_{-0.7}^{+0.2} \times 10^{45} \mathrm{erg} \mathrm{s}^{-1}$ of RX J1347 estimated in this work, overlayed onto the values measured for the mini-halo sample (Gitti et al. 2004). We note that RX J1347 follows nicely the observed trend, resulting one of the most powerful objects in the sample. This gives support to the possible classification of its diffuse radio source as a mini-halo triggered by the central cooling flow. However, the excess and elongation of the radio surface brightness coincident with the position of the hot subclump detected in X-rays (Figs. 4 and 5) might indicate that, besides the energy supplied by the central cooling flow, additional energy for the electron re-acceleration could be provided by the submerger. From this analysis we thus get indications that cluster mergers and cooling flows may act simultaneously in powering

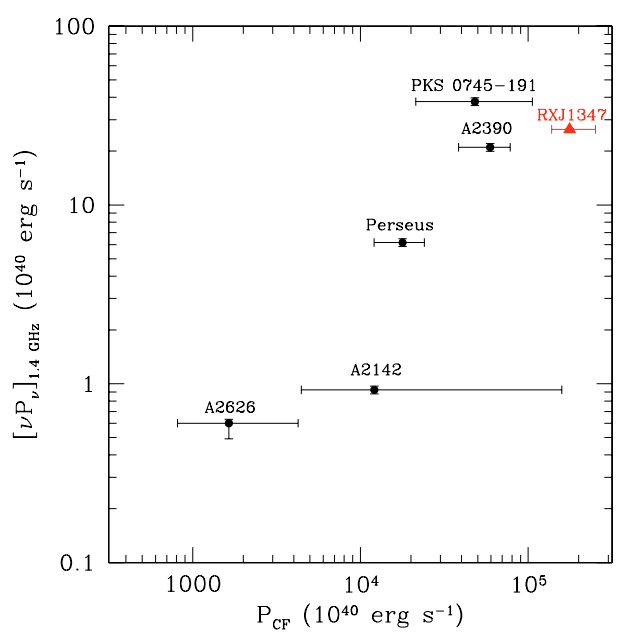

Fig. 6. Integrated radio power at $1.4 \mathrm{GHz},\left[v P_{v}\right]_{1.4 \mathrm{GHz}}$, vs. cooling flow power, $P_{\mathrm{CF}}=\dot{M} k T / \mu m_{\mathrm{p}}$, for the mini-halo clusters in Gitti et al. (2004). The triangle represents RX $\mathrm{J} 1347$ values estimated in this work $\left(P_{\mathrm{CF}}\right.$ from XMM-Newton data taken from Gitti \& Schindler 2004).

diffuse radio emission in the rare and peculiar clusters in which they coexist.

Further theoretical and observational studies are currently in progress and will give a more definite answer regarding the nature of the diffuse emission in RX J1347. In a more general context, we are also carrying out a project aimed at selecting and studying a large sample of radio mini-halos in order to further investigate their origin and their connection with giant radio halos.

Acknowledgements. We thank the anonymous referee for useful comments. We thank T. Erben for providing the optical image of the cluster. M.G. thanks D. Dallacasa for his helpful advises during the radio data analysis. C.F. acknowledges financial support of Austrian Science Foundation (FWF) through grant number P18523, and TirolerWissenschaftsfonds (TWF) through grant number UNI-0404/156.

\section{References}

Allen, S. W., Schmidt, R. W., \& Fabian, A. C. 2002, MNRAS, 335, 256 Blasi, P., \& Colafrancesco, S. 1999, Astropart. Phys., 12, 169 Blasi, P., Gabici, S., \& Brunetti, G. 2007, Int. J. Mod. Phys. A, 22, 681 Brunetti, G. 2003, ASP Conf. Ser., 301, 349

Brunetti, G., Setti, G., Feretti, L., \& Giovannini, G. 2001, MNRAS, 320, 365 Cohen, J. H., \& Kneib, J. 2002, ApJ, 573, 524

Condon, J. J., Cotton, W. D., Greisen, E. W., et al. 1998, AJ, 115, 1693

Fabian, A. C. 1994, ARA\&A, 32, 277

Feretti, L., \& Giovannini, G. 2007, [arXiv: astro-ph/0703494]

Gitti, M., Brunetti, G., \& Setti, G. 2002, A\&A, 386, 456

Gitti, M., Brunetti, G., Feretti, L., \& Setti, G. 2004, A\&A, 417, 1

Gitti, M., \& Schindler, S. 2004, A\&A, 427, 9

Gitti, M., Piffaretti, R., \& Schindler, S. 2007, A\&A, submitted

Gomez, P. L., Pinkney, J., Burns, J. O., et al. 1997, ApJ, 474, 580

Komatsu, E., Kitayama, T., Suto, Y., et al. 1999, ApJL, 516, 1

Komatsu, E., Matsuo, H., Kitayama, T., et al. 2001, PASJ, 53, 57

Peterson, J. R., \& Fabian, A. C. 2006, Phys. Rep., 427, 1

Pfrommer, C., \& Enßlin, T. 2004, A\&A, 413, 17

Pointecouteau, E., Giard, M., Benoit, A., et al. 1999, ApJ, 519, L115

Pointecouteau, E., Giard, M., Benoit, A., et al. 2001, ApJ, 552, 42

Schindler, S., Guzzo. L., Ebeling, H., et al. 1995, A\&A, 299, 9 